\title{
Trattamento avanzato delle acque reflue urbane mediante bioreattori a membrane autoformanti per il controllo dei contaminanti emergenti
}

\author{
Vincenza Vassallo ${ }^{1}$ \\ ${ }^{1}$ Affiliation not available
}

\begin{abstract}
Lo scarico di acque reflue trattate nei corpi idrici ricettori o il loro riutilizzo rende necessari sia gli interventi sugli impianti di depurazione esistenti sia lo sviluppo di nuove tecnologie innovative ed ecosostenibili in modo da assicurare maggiori efficienze depurative e rimuovere i contaminanti emergenti intercettati nelle acque reflue. Questi "contaminanti emergenti" si possono classificare secondo il loro uso e/o scopo; tra questi, quelli maggiormente intercettati nelle acque reflue sono i farmaci come antibiotici, antinfiammatori, ormoni naturali e sintetici. Gli attuali impianti di depurazione, purtroppo, non sono in grado di rimuovere questi composti e, quindi, vengono immessi nell'ambiente attraverso differenti modi come gli effluenti dei depuratori. Per ridurre al massimo l'impatto causato dai contaminanti emergenti, facciamo riferimento a delle moderne tecnologie: i bioreattori a membrana MBR, che derivano dalla combinazione dei processi di degradazione biologica a fanghi attivi e di filtrazione su membrana; i bioreattori a membrana elettricamente potenziati eMBR, che combinano i processi di biodegradazione, i processi elettrochimici e la filtrazione su membrana in un unico sistema, che permette di ottenere un effluente di qualità maggiore rispetto ai tradizionali MBR.
\end{abstract}




\section{Introduzione}

I bioreattori a membrana (MBR), derivanti dalla combinazione dei processi di degradazione biologica a fanghi attivi e di filtrazione a membrana, trovano oggi sempre più maggiore applicazione per il trattamento di reflui civili e industriali grazie all'elevata qualità degli effluenti trattati, che rispettano i limiti imposti dalle normative vigenti per lo scarico nei corsi idrici ricettori e il riutilizzo delle acque per scopi industriali o agricoli. Altro vantaggio di questa innovativa tecnica è rappresentato dal contenuto ingombro planimetrico rispetto ai processi convenzionali a fanghi attivi.

I bioreattori a membrana si sono anche dimostrati capaci di riuscire a trattare con successo i contaminanti emergenti (farmaci, pesticidi, droghe, microplastiche) che sempre con maggiore frequenza si riscontrano nelle acque reflue.

La progettazione di impianti a scala reale resta ancora piuttosto empirica a causa delle complesse interazioni che avvengono tra la biomassa e le membrane filtranti: ciò si traduce in maggiori costi d'investimento ed operativi, rispetto agli impianti convenzionali a fanghi attivi, principalmente correlati al contenimento del fouling delle membrane. L'attenzione del mondo scientifico è oggi pertanto volta prevalentemente alla caratterizzazione del fouling e alla definizione di strategie operative che siano in grado di minimizzarne la formazione nel tempo.

Negli ultimi anni la ricerca scientifica ha proposto nuove soluzioni, come l'applicazione di processi elettrochimici, che ha dimostrato ottime capacità nella mitigazione del fouling e nell'incremento delle efficienze di depurazione. Altra soluzione proposta, soprattutto per i paesi in via di sviluppo, è rappresenta dall'utilizzo di membrane innovative a basso costo ottenute anche con materiale di scarto.

Tuttavia, ad oggi nessun studio riporta la combinazione di queste due innovative tecniche di depurazione. 


\section{Bioreattori a membrana}

La tecnologia MBR ha attirato notevoli attenzioni, soprattutto per quanto riguarda il trattamento delle acque reflue, in quanto abbinano all'alta qualità dell'effluente prodotto un minore ingombro planimetrico. Tuttavia, l'applicazione su larga scala dei bioreattori a membrane è ostacolata da diversi problemi tra cui quello principale riguardante la formazione del fouling di membrana che causa una diminuzione del flusso di permeato; di conseguenza, i costi operativi già molto elevati dei processi MBR sono aumentati con la necessità di pulire o sostituire le membrane, il cui costo è già molto elevato. Per questo motivo sono state introdotte diverse tecniche e strategie, per la mitigazione del fouling ${ }^{1}$. Considerando che le tipologie di membrane, le caratteristiche e le dimensioni dei pori sono dei parametri fondamentali per il controllo del fouling e che, sia i costi capitali che di gestione degli impianti MBR sono elevati e devono essere ridotti drasticamente al fine di aumentare la loro competitività nel campo del trattamento delle acque reflue, negli ultimi anni, l'attenzione è stata rivolta verso l'utilizzo di membrane a basso costo (tessuti filtranti, mesh) ed autoformanti.

\section{Membrane dinamiche autoformanti}

In alternativa ai sistemi MBR convenzionali, è stata sviluppata una nuova tecnologia, che prevede la formazione e l'utilizzo delle membrane dinamiche auto-formanti all'interno del bioreattore (self-forming dynamic membrane bioreactor, SF-DMBR); “i bioreattori a membrane autoformanti presentano diversi vantaggi quali: facile controllo del fouling, bassi costi dei materiali utilizzati per le membrane, alto flusso di permeato, basso fabbisogno di energia ed elevata capacità di trattamento" ${ }^{2}$. 
Le membrane dinamiche possono essere classificate in due gruppi:

1. Membrane autoformanti (self-forming dynamic membrane SFDM);

2. Membrane pre-rivestite (precoated dynamic membrane PDM);

Le membrane dinamiche autoformanti sono generate dalle sostanze presenti nella miscela liquida che durante la filtrazione si depositano sulla superficie di supporto.

Le membrane pre-rivestite, denominate anche membrane FIP (formed in place), sono generate facendo filtrare sulla superficie di supporto, generalmente un materiale poroso, una soluzione di una o più componenti colloidali specifici; le membrane dinamiche pre-rivestite, a loro volta sono suddivise in due gruppi: membrane additive singole e composite ${ }^{3}$. Le prime sono formate da un solo componente o reagente e in un'unica fase mentre le membrane composite sono prodotte da un processo a più fasi. In un bioreattore a membrana dinamica DMBR (dynamic membrane reactor) si va a sostituire la membrana di separazione con una membrana dinamica autoformante. La formazione della membrana dinamica è un processo complesso che include molti meccanismi fisici, chimici e biologici come il deposito di particelle, il blocco dei pori e la formazione di uno strato di "cake" ${ }^{4}$. La membrana viene divisa in tre fasi (Figura 1):

1. strato di substrato: costituito da particelle con diametro di $0,1 \mathrm{~mm}$;

2. strato di separazione: costituito da particelle di fango la cui dimensione è ridotta dall'interno verso l'esterno e, la capacità di separazione di questo stato si può paragonare alla micromembrana;

3. strato di incrostazione costituito da particella di fango, soluti e colloidi. 


\section{Membrane elettricamente potenziate}

I bioreattori a membrana elettricamente potenziati eMBR combinano i processi di biodegradazione biologica, i processi elettrochimici e la filtrazione su membrana in un unico sistema che permette di raggiungere risulti migliori rispetto ai tradizionali MBR. Alcuni studi condotti da ${ }^{5} \mathrm{e}^{6}$, hanno dimostrato che l'applicazione di un campo elettrico nei bioreattori a membrana (eMBR) può ridurre il fouling in modo efficace. Il metodo introdotto da ${ }^{7}$ induce meccanismi elettrochimici ad aiutare a degradare gli inquinanti e controllare la mobilità e la deposizione di agenti contaminanti sulla superficie della membrana. Tra i processi elettrochimici (elettrocoagulazione, elettrosmosi, elettroforesi), l'elettrocoagulazione è il meccanismo principale che influenza la rimozione di composti organici con un elevato potenziale di incrostazione dalle acque reflue; implica la generazione in sito di coagulanti dalla dissoluzione elettrochimica di anodi immersi (ferro o alluminio), (Figura 2, c). Durante l'elettrocoagulazione, a seconda del $\mathrm{pH}$, vengono prodotte varie specie metalliche che reagiscono con gli inquinanti portando alla destabilizzazione e all'aggregazione delle particelle sospese e alla precipitazione e adsorbimento dei contaminanti disciolti ${ }^{8}$. La tensione applicata consente ai contaminanti caricati negativamente come i fanghi attivi o polimeri secreti di spostarsi verso l'elettrodo opposto e allontanarsi dalla membrana attraverso un movimento elettroforetico (Figura 2, a), ${ }^{9}$. Di conseguenza, la forza elettrosmotica spinge la rimozione di acqua legata dal doppio strato elettrico dei microbi che riduce la resistenza specifica dei fanghi alla filtrazione migliorando quindi il controllo del fouling (Figura 2, b), ${ }^{10}$

Le principali configurazioni eMBR applicate nel trattamento delle acque reflue si possono classificare in:

1. Unità elettrochimica esterna (di solito unità di elettrocoagulazione) che precede un bioreattore a membrana sommersa EC-SMBR (Figura 3, (a)); 


\section{Elettro-bioreattore a membrana sommersa SMEBR (Figura 3, (b))}

L'EC-SMBR è stato utilizzato ${ }^{11},{ }^{12}$ per il trattamento delle acque reflue urbane, delle acque grigie e delle acque reflue di conceria. In questo caso, l'influsso viene prima trattato mediante il processo di elettrocoagulazione in un'unità separata prima della degradazione biologica e della filtrazione su membrana in un bioreattore a membrana sommersa convenzionale (SMBR). Nei SMEBR, i processi elettrochimici sono integrati con il bioreattore a membrana mediante l'aggiunta di elettrodi sacrificali all'interno di SMBR. Le prime acque reflue attraversano la zona di trattamento biologico per la biodegradazione, cioè tra la parete del reattore e l'anodo, quindi attraverso la zona elettrica (gli elettrodi) per subire alcuni processi elettrochimici e un ulteriore degrado e, infine, vengono filtrati attraverso il modulo di membrana per la rimozione dei solidi 13

\section{Conclusioni}

Gli MBR presentano molti vantaggi, infatti, abbiamo dimostrato che questi riescono a produrre un effluente di alta qualità grazie alla capacità delle membrane di effettuare una vera e propria azione stracciante, la produzione di fanghi dimezzata e un ingombro planimetrico ridotto drasticamente grazie all'assenza del sedimentatore secondario. Mentre il principale svantaggio degli MBR è rappresentato dal fouling della membrana (sporcamento) che comporta un incremento non trascurabile dei costi di investimento e di gestione di questi impianti e, la riduzione dell'efficienza della filtrazione. Il fouling è influenzato dal tipo di materiale e dalla configurazione delle membrane filtranti, ma anche dalla miscela liquida, ricca di colloidi, fango biologico. Oggi, a causa di questo, vengono messe in atto diverse strategie atte a mitigare il fouling di membrana, tra queste l'uso delle SFDM, ovvero le membrane dinamiche autoformanti che rappresentano una efficiente soluzione al problema. I bioreattori a membrane autoformanti che presentano diversi vantaggi descritti precedentemente, sono membrane generate dalle sostanze presenti nella miscela liquida che durante la filtrazione si depositano sulla superficie di supporto, costituita da materiali a basso costo come 
dacron o nylon, incrementando le capacità depurative del sistema. Mentre, gli ESFDM, ovvero le membrane elettricamente potenziate, si basano sullo sviluppo di alcuni fenomeni elettrochimici che favoriscono la riduzione del fouling e, al contempo, garantiscono una maggiore capacità di rimozione degli inquinanti sia convenzionali che emergenti. Tra i diversi processi che si sviluppano è possibile distinguere l'elettrocoagulazione, l'elettrosmosi e l'elettroforesi. Tuttavia, gli effetti del campo elettrico fanno sì che le specie batteriche subiscano un forte shock che inibisce il loro metabolismo.

\section{References}

1.Liu, H., Yang, C., Pu, W. \& Zhang, J. Formation mechanism and structure of dynamic membrane in the dynamic membrane bioreactor. Chemical Engineering Journal 148, 290-295 (2009).

2.Xiong, J., Fu, D. \& Singh, R. P. Self-adaptive dynamic membrane module with a high flux and stable operation for the municipal wastewater treatment. Journal of Membrane Science 471, 308-318 (2014).

3.Ye, M. et al.. Study on the suitable thickness of a PAC-precoated dynamic membrane coupled with a bioreactor for municipal wastewater treatment. Desalination 194, 108-120 (2006).

4.Bae, T. \& Tak, T. Interpretation of fouling characteristics of ultrafiltration membranes during the filtration of membrane bioreactor mixed liquor. Journal of Membrane Science 264, 151-160 (2005).

5.Chu, D. et al.. Biotype Status and Distribution of IBemisia tabaci/I (Hemiptera: Aleyrodidae) in Shandong Province of China Based on Mitochondrial DNA Markers. Environmental Entomology 
36, 1290-1295 (2007).

6.Liu, L., Liu, J., Gao, B. \& Yang, F. Minute electric field reduced membrane fouling and improved performance of membrane bioreactor. Separation and Purification Technology 86, 106-112 (2012).

7.Bani-Melhem, K. \& Elektorowicz, M. Performance of the submerged membrane electrobioreactor (SMEBR) with iron electrodes for wastewater treatment and fouling reduction. Journal of Membrane Science 379, 434-439 (2011).

8.Giwa, A., Daer, S., Ahmed, I., Marpu, P. R. \& Hasan, S. W. Experimental investigation and artificial neural networks ANNs modeling of electrically-enhanced membrane bioreactor for wastewater treatment. Journal of Water Process Engineering 11, 88-97 (2016).

9.Chen, J., Yang, C., Zhou, J. \& Wang, X. Study of the influence of the electric field on membrane flux of a new type of membrane bioreactor. Chemical Engineering Journal 128, 177-180 (2007).

10.Ibeid, S., Elektorowicz, M. \& Oleszkiewicz, J. A. Modification of activated sludge properties caused by application of continuous and intermittent current. Water Research 47, 903-910 (2013).

11.Kim, H., Jang, H., Kim, H., Lee, D. \& Chung, T. Effect of an electro phosphorous removal process on phosphorous removal and membrane permeability in a pilot-scale MBR. Desalination 250, 629-633 (2010).

12.Bani-Melhem, K. \& Smith, E. Grey water treatment by a continuous process of an electrocoagulation unit and a submerged membrane bioreactor system. Chemical Engineering Journal 198-199, 201-210 (2012).

13.Hasan, S., Elektorowicz, M. \& Oleszkiewicz, J. Start-up period investigation of pilot-scale submerged membrane electro-bioreactor (SMEBR) treating raw municipal wastewater. Chemosphere 97, 71-77 (2014). 


\section{Figure Captions}

Figure 1. Impianti di trattamento

Figure 2. Struttura della membrana dinamica, adatta da ${ }^{1}$

Figure 3. Meccanismi elettrochimici che si verificano nel funzionamento dell'elettro MBR (eMBR): elettroforesi (a), elettrosmosi (b), elettrocoagulazione (c), adatta da ${ }^{9}$

Figure 4. Unit@x@x tempboxaa@spacefactor $18 a \Gamma @$ spacefactorelettrochimicaesternacheprecedeunbior $\operatorname{SMBR}(a)$, elettrobioreattoreamembranasommersaSMEBR(b), adattada ${ }^{\Omega-12}$ 


\section{Figures}

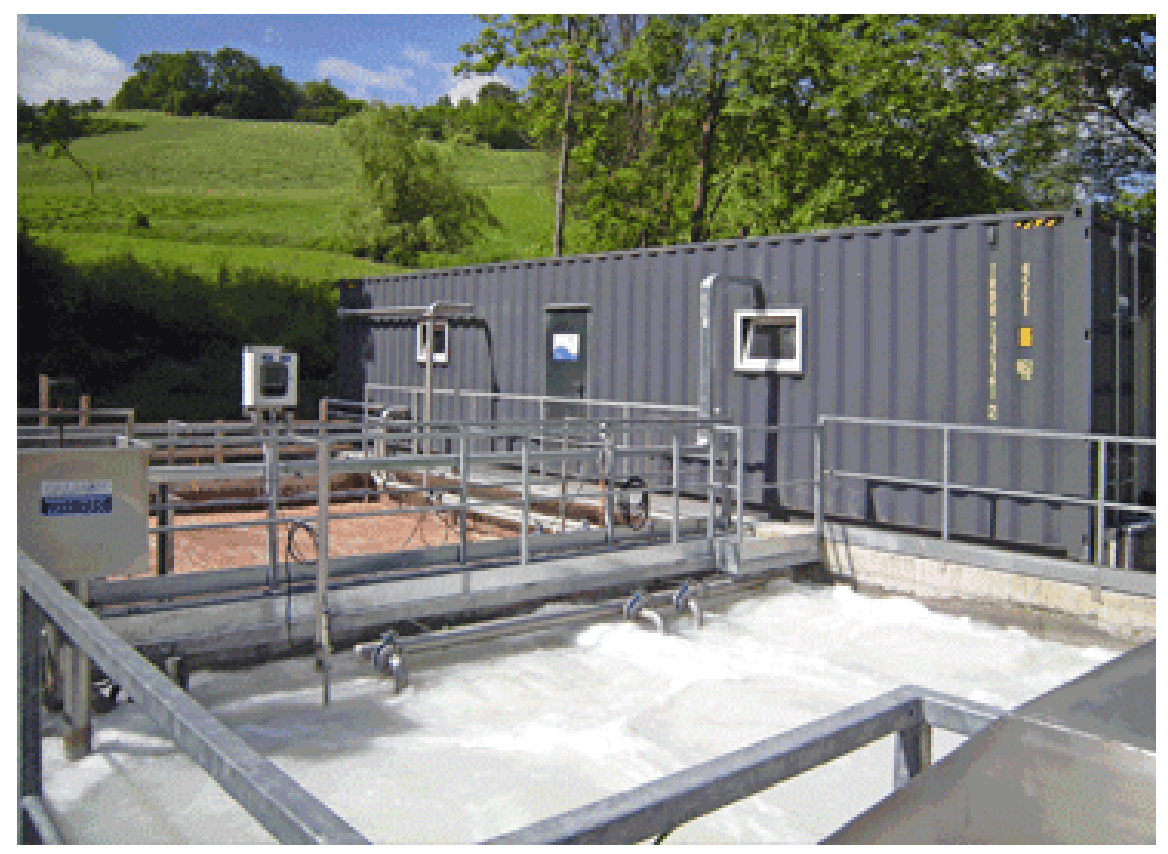

Figure 1: Impianti di trattamento

Figure 2: Struttura della membrana dinamica, adatta da ${ }^{1}$

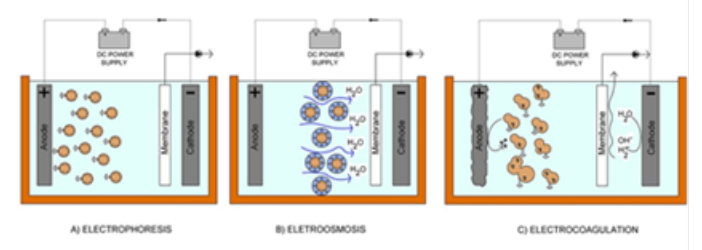

Figure 3: Meccanismi elettrochimici che si verificano nel funzionamento dell'elettro MBR (eMBR): elettroforesi (a), elettrosmosi (b), elettrocoagulazione (c), adatta da ${ }^{9}$ 


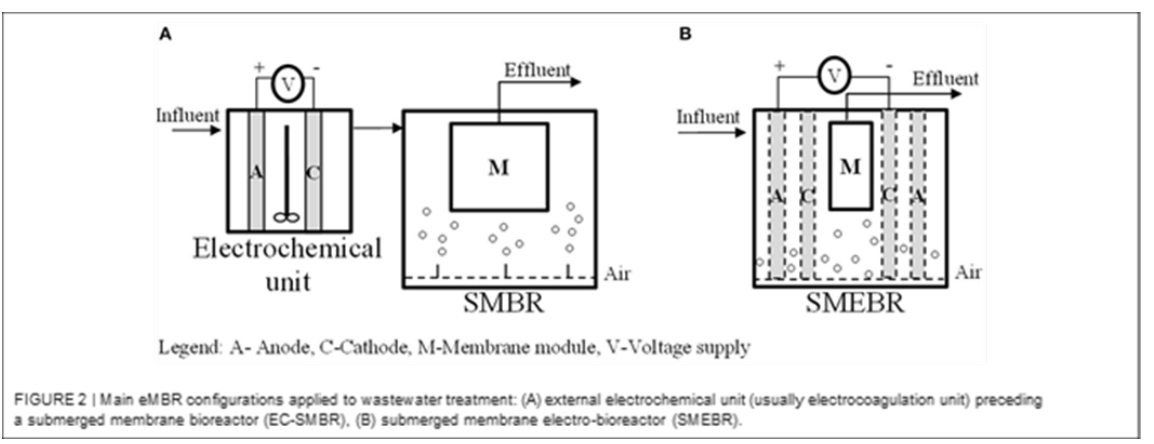

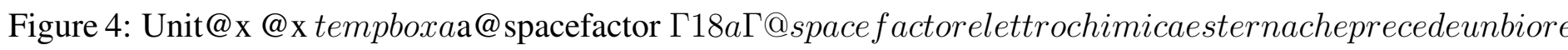
$S M B R(a)$, elettrobioreattoreamembranasommersaSMEBR(b), adattada ${ }^{\Omega-12}$ 